\title{
MicroRNA-512-3p is upregulated, and promotes proliferation and cell cycle progression, in prostate cancer cells
}

\author{
ZHIGANG RAO ${ }^{1}$, ZIQI HE ${ }^{2}$, YI HE ${ }^{3}$, ZONGHUA GUO ${ }^{1}$, DONGBO KONG ${ }^{1}$ and JUFANG LIU ${ }^{3}$ \\ ${ }^{1}$ Department of Urology, Xianning Central Hospital, The First Affiliated Hospital of Hubei University of Science and Technology, \\ Xianning, Hubei 437000; ${ }^{2}$ Department of Urology, The First Affiliated Hospital of Guangxi Medical University, Nanning, \\ Guangxi 530000; ${ }^{3}$ Department of Obstetrics and Gynecology, Xianning Central Hospital, The First Affiliated Hospital of \\ Hubei University of Science and Technology, Xianning, Hubei 437000, P.R. China
}

Received December 14, 2016; Accepted July 7, 2017

DOI: $10.3892 / \mathrm{mmr} .2017 .7844$

\begin{abstract}
Prostate cancer (PCa) is the most commonly diagnosed cancer in males worldwide. MicroRNAs (miRNAs/miRs) are small non-coding RNAs that participate in the regulation of various biological processes by regulating post-transcriptional gene expression. However, whether dysregulation of miRNA expression may be associated with the carcinogenesis of $\mathrm{PCa}$ remains to be elucidated. The present study identified differentially expressed miRNAs in PCa by analyzing two publicly available gene expression datasets, GSE14857 and GSE21036. The results demonstrated that miR-512-3p was significantly upregulated in $\mathrm{PCa}$. Furthermore, the present study explored the molecular functions of miR-512-3p in PCa, and demonstrated that overexpression of miR-512-3p promoted $\mathrm{PCa}$ cell proliferation and reduced $\mathrm{G}_{1}$ phase cell cycle arrest in $\mathrm{PCa}$. These results indicated that miR-512-3p may act as an oncogene in PCa. To the best of our knowledge, this is the first study revealed the molecular functions of miR-512-3p in PCa. To obtain valuable insights into the potential mechanisms of miR-512-3p, bioinformatics analyses were performed to identify the targets of miR-512-3p. Kyoto Encyclopedia of Genes and Genomes pathway and Gene Ontology category analyses revealed that miR-512-3p may be associated with the mitogen-activated protein kinase signaling pathway and numerous biological processes, including cell adhesion, cell proliferation, cell cycle and apoptosis. These results suggested that miR-512-3p may be considered a potential diagnostic and therapeutic target of $\mathrm{PCa}$.
\end{abstract}

Correspondence to: Dr Jufang Liu, Department of Obstetrics and Gynecology, Xianning Central Hospital, The First Affiliated Hospital of Hubei University of Science and Technology, 88 Jin Gui Road, Xianan, Xianning, Hubei 437000, P.R. China

E-mail: dagang_group@outlook.com

Key words: prostate cancer, microRNA-512-3p, proliferation, cell cycle

\section{Introduction}

Prostate cancer $(\mathrm{PCa})$ is one of the most common types of cancer, and the leading cause of cancer-associated mortality in men worldwide $(1,2)$. The molecular mechanisms that underlie the tumorigenesis, progression and metastasis of PCa remain unclear, regardless of a large number of research studies. Noncoding RNAs have been reported to serve pivotal roles in the pathogenesis of numerous types of cancer via regulating the diversity of biological processes. Therefore, identifying the noncoding RNAs associated with PCa may provide a novel insight into the mechanisms underlying PCa carcinogenesis (3-6).

MicroRNAs (miRNAs/miRs) are small post-transcriptional regulatory noncoding RNAs, 20-22 nt in length. Previous studies have reported the aberrant expression of miRNAs in numerous types of human malignancy, including breast and lung cancer, and PCa (7-11). In PCa, numerous dysregulated miRNAs, inducing miR-27a (12,13), miR-135a (14), miR-186 (15), miR-4638-5p (16), miR-124 (17-19) and miR-320 (20,21), have been reported to regulate cell growth, apoptosis, migration and invasion. These findings indicated that dysregulation of miRNA expression may be associated with carcinogenesis of PCa.

miR-512-3p has been reported to act as a tumor suppressor in hepatocellular carcinoma (22) and lung adenocarcinoma (23). In addition, miR-512-3p has also been revealed to be upregulated in non-small cell lung cancer (NSCLC) A549 cells following retinoic acid (RA) treatment, and has been demonstrated to inhibit the adhesion, migration and invasion of NSCLC cells (23). However, the role of miR-512-3p in PCa remains poorly understood and therefore requires further investigation.

The present study identified differentially expressed miRNAs in PCa, by analyzing two publicly available gene expression datasets, GSE14857 (24) and GSE21036 (25). Furthermore, the molecular functions of miR-512-3p in PCa were investigated, in order to identify their potential roles in the carcinogenesis of $\mathrm{PCa}$.

\section{Materials and methods}

miRNA profile data collection. miRNA profile datasets (GSE14857 and GSE21036) were collected from the Gene Expression Omnibus database (www.ncbi.nlm.nih.gov/gds). 
Comparison of the miRNA profiles between PCa samples and normal tissue samples was performed with limma package in R software using raw microarray data. Significantly differentially expressed miRNAs were identified with thresholds of $\mid \log \mathrm{FCl}>1.0$ and $\mathrm{P}<0.05$.

Cell culture. LNCaP cells were purchased from the American Type Culture Collection (Manassas, VA, USA). PC-3 and 22RV1 cells, and the noncancerous prostatic cell line WPMY-1, were obtained from the Cell Bank of Chinese Academy of Sciences (Shanghai, China). All cell lines were confirmed by short tandem repeat analysis. The four cell lines were cultured in Ham's F12K media (Invitrogen; Thermo Fisher Scientific, Inc., Waltham, MA, USA) supplemented with $10 \%$ (vol/vol) fetal bovine serum (cat. no. 10099141M; Gibco; Thermo Fisher Scientific, Inc.), at $37^{\circ} \mathrm{C}$ in a humidified atmosphere containing $5 \% \mathrm{CO}_{2}$.

Cell transfection. The synthetic miR-512-3p mimics and a scrambled control miRNA [miR-negative control (NC)] were purchased from Shanghai GenePharma Co., Ltd. (Shanghai, China). The sequences were as follows: miR-512-3p mimics, 5'-AAGUGCUGUCAUAGCUGAGGUC-3' (sense) and 5'-CCUCAGCUAUGACAGCACUUUU-3' (antisense); NC mimics, 5'-UUCUCCGAACGUGUCACGUTT-3' (sense) and 5'-ACGUGACACGUUCGGAGAATT-3' (antisense). PCa cells were seeded at $3 \times 10^{5}$ cells/wells in 6 -well plates and were incubated at $37^{\circ} \mathrm{C}$ in a humidified atmosphere containing $5 \% \mathrm{CO}_{2}$ overnight. Subsequently, transfection with the miR-512-3p mimic or miR-NC was performed using Lipofectamine 2000 transfection reagent (Invitrogen; Thermo Fisher Scientific, Inc.). The cells were transfected with $300 \mathrm{nmol}$ miRNA according to the manufacturer's protocol. Total RNA was extracted from the cells $48 \mathrm{~h}$ post-transfection and western blotting was also performed.

RNA extraction and reverse transcription-quantitative polymerase chain reaction $(R T-q P C R)$. Total RNA, which was used for RT-qPCR analysis, was extracted from the cells using TRIzol reagent (Invitrogen; Thermo Fisher Scientific, Inc.) according to the manufacturer's protocol. RT was performed using the PrimeScript ${ }^{\mathrm{TM}}$ RT reagent kit (Takara Bio, Inc., Otsu, Japan) according to the manufacturer's protocol. The sequence of the miR-512-3p-specific RT primer was 5'-GTC GTATCCAGTGCAGGGTCCGAGGTATTCGCACTGGAT ACGACGACCTC-3'. To analyze miRNA expression, RT-qPCR was performed using SYBR-Green Reagents (Bio-Rad Laboratories, Inc., Hercules, CA, USA) on a LightCycler 480 system (Roche Diagnostics, Basel, Switzerland). The expression levels of miR-512-3p were normalized to U6. The PCR primers for mature miR-512-3p, Rho family GTPase 3 (RND3), MX dynamin like GTPase 1 (MXI), mitofusin 2 (MFN2), forkhead box O1 (FOXO1), RNA binding motif protein38 (RBM38), transforming coiled-coil containing protein 1 (TACC1) and U6 were as follows: miR-512-3p forward, 5'-CGG CGGCACTCAGCCTTGAGGG-3' and reverse, 5'-GTGCAG GGTCCGAGGT-3'; RND3 forward, 5'-AAAAACTGCGCT GCTCCAT-3' and reverse, 5'-TCAAAACTGGCCGTGTAA TTC-3'; MXI1 forward, 5'-CATGGAGCGGGTGAAGAT-3' and reverse, 5'-ATGAAGAGGCGTAGCCATGT-3'; MFN2 forward, 5'-TGCCTCAGAGCCCGAGTA-3' and reverse,
5'-CTGGTACAACGCTCCATGTG-3'; FOXO1 forward, 5'-AAGGGTGACAGCAACAGCTC-3' and reverse, 5'-TTC CTTCATTCTGCACACGA-3'; RBM38 forward, 5'-TTGATC CAGCGGACTTACG-3' and reverse, 5'-AATGTAGGGCGA GGACAGC-3'; TACC1 forward, 5'-GCGAAATGGACGTGG TCT-3' and reverse, 5'-CACCTTACAGCCACTCCTGAA-3'; and U6 forward, 5'-CGCTTCGGCAGCACATATACTAA-3' and reverse 5'-TATGGAACGCTTCACGAATTTGC-3'. The results were normalized to those of $\beta$-actin or U6 as the internal control to estimate the different expression of genes. Relative mRNA and miRNA expression was calculated using the $2^{-\Delta \Delta \mathrm{Cq}}$ method (26). Each sample was assayed in triplicate to ensure quantitative accuracy.

Western blot analysis. Cells were lysed in radioimmunoprecipitation assay buffer (Boston Bioproducts, Inc., Ashland, MA, USA) supplemented with cOmplete ${ }^{\mathrm{TM}}$, EDTA-free Protease Inhibitors (Roche Diagnostics) and phenylmethylsulfonyl fluoride (Calbiochem; EMD Millipore, Billerica, MA, USA). The protein concentration was determined using the Pierce ${ }^{\mathrm{TM}}$ Bicinchoninic acid Protein Assay (cat. no. 23222; Thermo Fisher Scientific, Inc.), in accordance with the manufacturer's instructions. A total of $30 \mu \mathrm{g} /$ lane protein was loaded and separated by $12 \%$ SDS-PAGE, which was then transferred to PVDF membranes. Membranes were blocked in Tris buffered saline with $0.05 \%$ Tween-20 containing 5\% non-fat dry milk at room temperature for $1 \mathrm{~h}$. Immunoblots were incubated overnight at $4^{\circ} \mathrm{C}$ with the following primary antibodies: Anti-p21 (1:1,000; cat. no. ab109520; Abcam, Cambridge, MA, USA) and anti- $\beta$-actin (1:3,000; cat. no. A1978; Sigma-Aldrich; Merck KGaA, Darmstadt, Germany). Subsequently, the blots were incubated at room temperature for $1 \mathrm{~h}$ with goat anti-mouse immunoglobulin (Ig)G-horseradish peroxidase (HRP)-conjugated and goat anti-rabbit IgG-HRP-conjugated secondary antibodies (1:4,000; cat. nos. A4416 and A6154, respectively; Sigma-Aldrich; Merck KGaA). An Electrochemiluminescence Plus kit (cat. no. RPN2132; GE Healthcare Life Sciences, Uppsala, Sweden) was used for visualization.

Cell proliferation assay. Cells were seeded into 96-well plates at 2,000 cells/well $6 \mathrm{~h}$ post-transfection. The Cell Counting kit-8 (CCK-8; Dojindo Molecular Technologies, Inc., Kumamoto, Japan) was used to detect relative cell proliferation for 4 days. Briefly, $10 \mu \mathrm{l} /$ well CCK- 8 agent was added to the cells, which were incubated for $2 \mathrm{~h}$ at $37^{\circ} \mathrm{C}$; subsequently, absorbance was measured at $450 \mathrm{~nm}$ using an ELx808 microplate reader (BioTek Instruments, Inc., Winooski, VT, USA).

Cell cycle analysis. Transfected LNCaP, 22Rv1 and PC-3 cells in the log phase of growth were collected and fixed in $0.03 \%$ Triton X-100 and propidium iodide (PI; $50 \mathrm{ng} / \mathrm{ml}$ ) at room temperature for $15 \mathrm{~min}, 48 \mathrm{~h}$ post-transfection. For cell cycle analysis, the transfected cells were examined using a FACSCalibur flow cytometer (BD Biosciences, San Jose, CA, USA) and were analyzed with ModFit version 4.1 software (Verity Software House, ME, USA). Each test was performed in triplicate.

Gene Ontology (GO) and Kyoto Encyclopedia of Genes and Genomes (KEGG) pathway analysis. The Molecule 
A
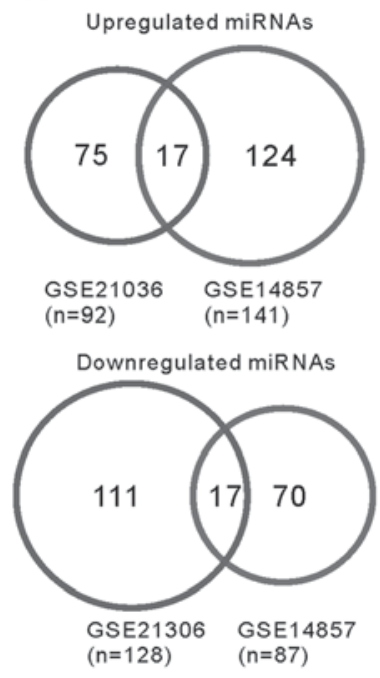

D

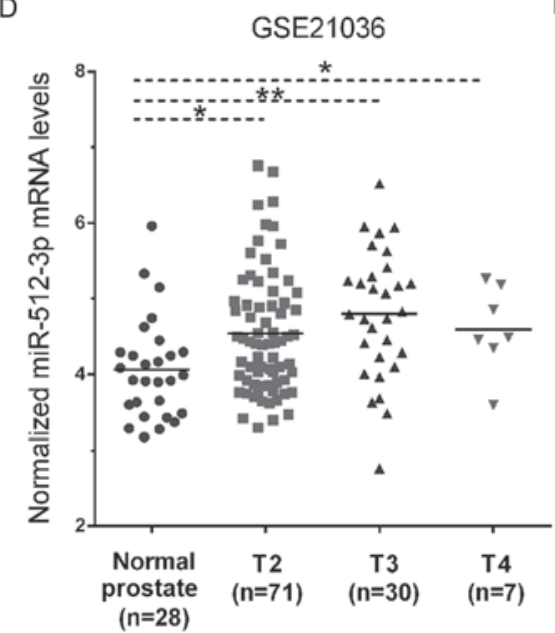

B

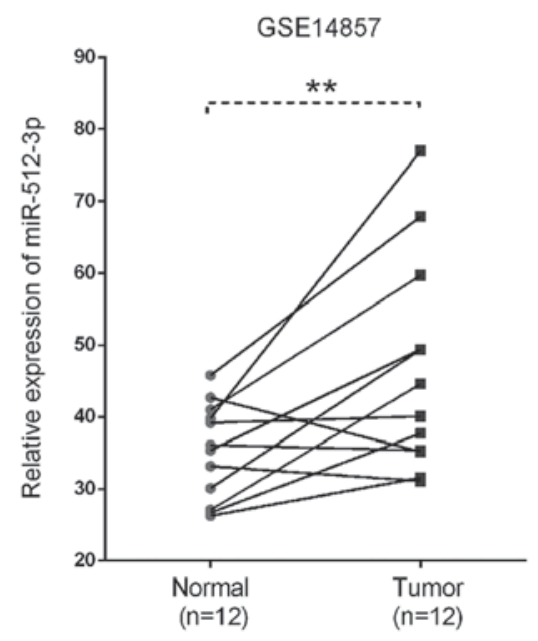

$\mathrm{E}$

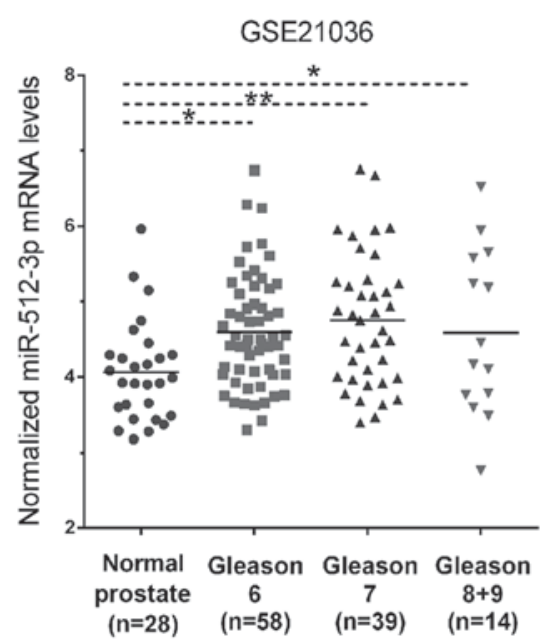

c

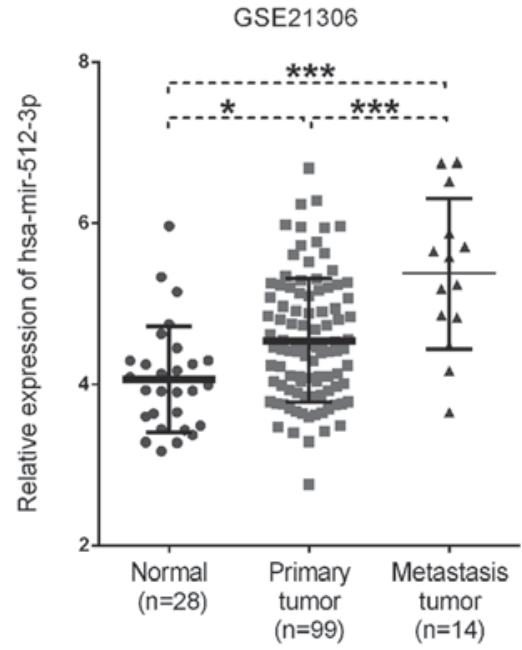

F

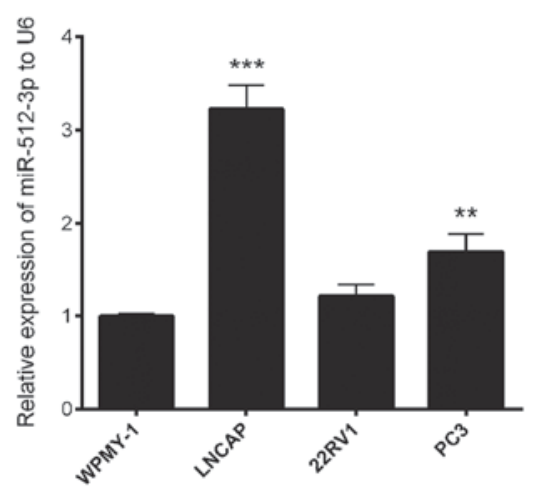

Figure 1. miR-512-3p is overexpressed in prostate cancer. (A-C) miR-512-3p was overexpressed in two publicly available gene expression datasets, GSE14857 and GSE21036. (D) miR-512-3p expression levels were overexpressed in patients with $\mathrm{T} 2(\mathrm{P}<0.05), \mathrm{T} 3(\mathrm{P}<0.01)$ and $\mathrm{T} 4(\mathrm{P}<0.05)$ prostate cancer compared with the normal controls. (E) Expression levels of miR-512-3p were upregulated in Gleason $6(\mathrm{P}<0.05)$, Gleason $7(\mathrm{P}<0.01)$ and Gleason $8(\mathrm{P}<0.05)$ prostate cancer when compared with the normal controls (all vs. NC). (F) Expression levels of miR-512-3p were upregulated in LNCaP $(\mathrm{P}<0.001)$ and $\mathrm{PC}-3(\mathrm{P}<0.01)$ when compared with the WPMY-1 normal prostate cell line (both vs. WPMY-1). ${ }^{*} \mathrm{P}<0.05,{ }^{* * *} \mathrm{P}<0.01$ and ${ }^{* * * *} \mathrm{P}<0.001$, as indicated. miR, microRNA; T2, intraprostatic localized tumors; $\mathrm{T} 3+\mathrm{T} 4$, invasive extraprostatic tumors.

Annotation System (MAS; version 3.0), provided by CapitalBio Corporation (Beijing, China; bioinfo.capitalbio.com/mas3/) was used to determine the biological roles of differentially expressed mRNAs. Gene functions were classified in to three subgroups: Biological process, cellular component and molecular function. The enriched GO terms were presented by enrichment scores. KEGG pathway analysis was carried out to determine the involvement of differentially expressed mRNAs in different biological pathways. The recommended hypergeometric-P-value used as the cut-off was $\mathrm{P}<0.05$.

Statistical analysis. Numerical data were presented as the mean \pm standard deviation of at least three determinations. Statistical comparisons between groups of normalized data were performed using an unpaired Student's t-test and SPSS v13.0 software (SPSS, Inc., Chicago, IL, USA) or a Mann-Whitney U-test according to the test condition. Statistical comparisons among multiple groups of normalized data were performed using one-way analysis of variance followed by a Dunnett's post hoc test. $\mathrm{P}<0.05$ was considered to indicate a statistically significant difference with a $95 \%$ confidence level.

\section{Results}

miR-512-3p is overexpressed in PCa. To identify the differentially expressed miRNAs in $\mathrm{PCa}$, two publicly available gene expression datasets, GSE14857 and GSE21036, were analyzed (Fig. 1A). A total of 17 miRNAs were downregulated and 17 miRNAs were overexpressed in $\mathrm{PCa}$ in both databases. The present study primarily focused on the 17 upregulated miRNAs (miR-106b, miR-93, miR-148a, miR-25, miR-375, miR-130b, miR-512-3p, miR-18a, miR-518c ${ }^{*}$, miR-7, miR-95, miR-96, miR-32, miR-663, miR-182, miR-183 and miR-153) as putative biomarkers. The majority of these miRNAs have been reported to be involved in the carcinogenesis of PCa (27-37); however, the molecular functions of miR-512-3p and miR-518 $\mathrm{c}^{*}$ in PCa remained unclear. 
A
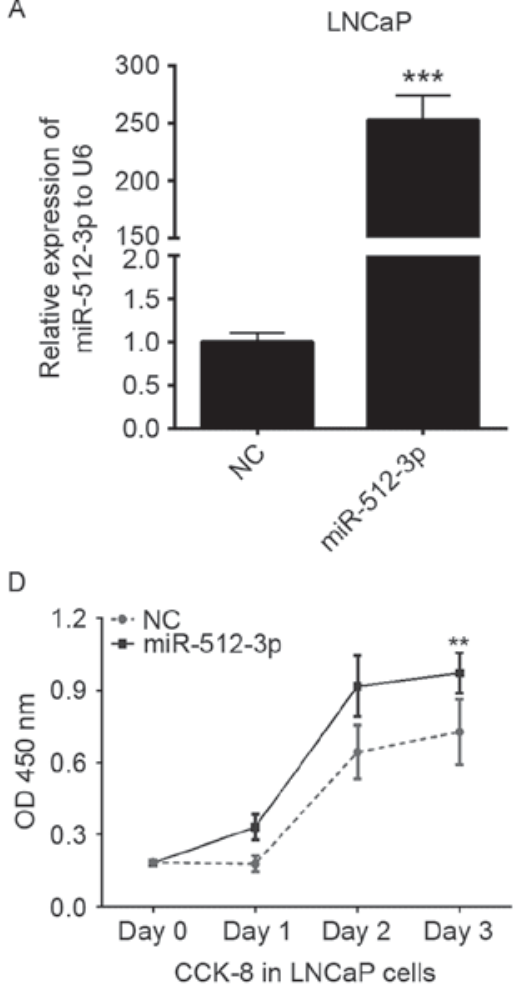

B

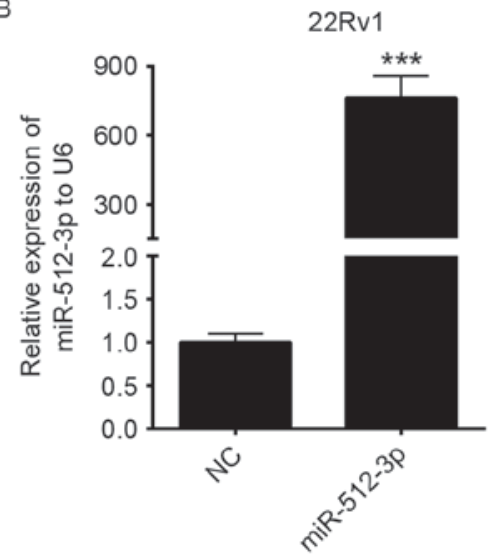

E

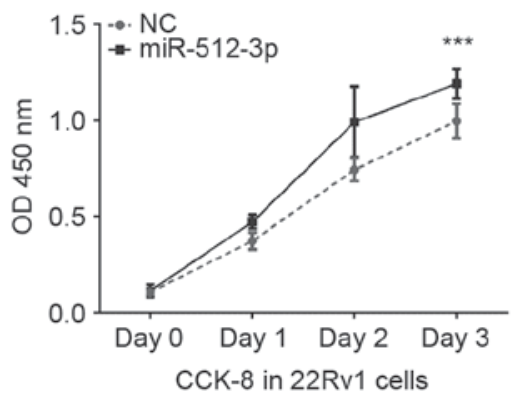

C

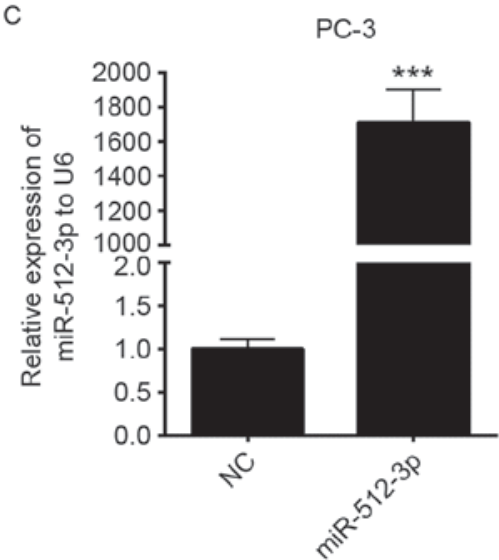

$\mathrm{F}$

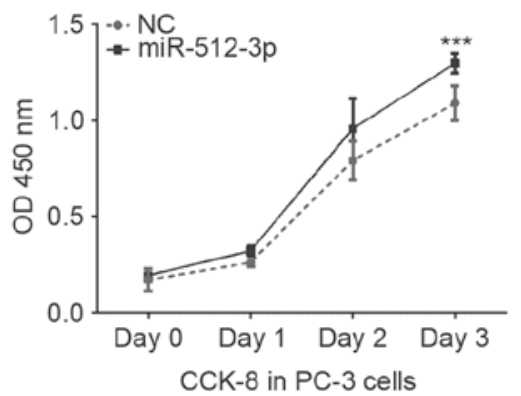

Figure 2. miR-512-3p promotes prostate cancer cell proliferation. miR-512-3p expression was significantly upregulated in (A) LNCaP, (B) 22Rv1 and (C) PC-3 prostate cancer cells following transfection with miR-512-3p mimics for $48 \mathrm{~h}$ using reverse transcription-quantitative polymerase chain reaction. Overexpression of miR-512-3p promoted (D) LNCaP, (E) 22Rv1 and (F) PC-3 prostate cancer cell proliferation via a CCK-8 assay. Data are presented as the mean \pm standard deviation $(\mathrm{n}=8) .{ }^{* *} \mathrm{P}<0.01$ and ${ }^{* * *} \mathrm{P}<0.001$ vs. NC. CCK-8, Cell Counting kit-8; miR, microRNA; NC, negative control; OD, optical density.

Analysis of the GSE14857 and GSE21036 datasets indicated that miR-512-3p expression was significantly upregulated in tumor samples compared with in normal samples $(\mathrm{P}<0.01$; Fig. 1B), and was overexpressed in metastatic samples compared with in primary tumor tissues $(\mathrm{P}<0.001$; Fig. $1 \mathrm{C})$. A clinical significance analysis of GSE21036 demonstrated that miR-512-3p expression levels were overexpressed in patients with T2 $(\mathrm{P}<0.05), \mathrm{T} 3(\mathrm{P}<0.01)$ and T4 $(\mathrm{P}<0.05)$ PCa compared with the normal controls (Fig. 1D). Subsequently, the patients in GSE21036 were categorized based on Gleason grades, and the results demonstrated that tissues from patients with Gleason grades 6, 7,8 and 9 PCa exhibited significantly higher levels of miR-512-3p compared with the matched normal tissues (Fig. 1E).

The present study also detected miR-512-3p expression in PCa cell lines. RT-qPCR was conducted to detect the expression levels of miR-512-3p in PCa cell lines LNCaP, 22Rv1 and PC-3, and in the noncancerous prostatic cells WPMY-1 cell line. The results demonstrated that miR-512-3p was upregulated in PCa cells (including LNCaP and PC-3; Fig. 1F). However, no significant upregulation of miR-512-3p was observed in 22Rv1 cells. These results were consistent with the previous findings in PCa and normal tissues.

Overexpression of miR-512-3p promotes $P C$ a cell proliferation. The present study aimed to explore the potential effects of miR-512-3p on the proliferation of PCa cells. Initially, the effects of a miR-512-3p mimic were determined on the expression of miR-512-3p. LNCaP, 22Rv1 and PC-3 cells were transfected with NC or miR-512-3p mimics. A total of
$48 \mathrm{~h}$ post-transfection, the expression levels of miR-512-3p were significantly increased in the miR-512-3p mimic group compared with the NC group ( $\mathrm{P}<0.001$; Fig. 2A-C). Subsequently, cell proliferation was investigated using a CCK-8 assay, overexpression of miR-512-3p significantly promoted the proliferation of LNCaP, 22Rvland PC-3 cells $(\mathrm{P}<0.01$ and $\mathrm{P}<0.001$; Fig. 2D-F $)$.

Overexpression of miR-512-3p prevents $G_{1}$ phase cell cycle arrest in vitro. The present study assessed the function of miR-512-3p on cell cycle progression in LNCaP, 22Rv1 and $\mathrm{PC}-3$ cells. Flow cytometric analysis revealed that overexpression of miR-512-3p in LNCaP and 22RV1 cells resulted in a significant increase in the proportion of cells in $\mathrm{S}$ phase and a decrease in the proportion of cells in G1 phase. However, overexpression of miR-512-3p in PC-3 cells decreased the proportion of cells in $\mathrm{S}$ phase and increased the proportion of cells in the $\mathrm{G} 2 / \mathrm{M}$ phase $(\mathrm{P}<0.05$; Fig. $3 \mathrm{~A}-\mathrm{C})$. In addition, a decrease in the protein expression levels of cell cycle inhibitor p21 was detected in cells overexpressing miR-512-3p (Fig. 3D). Downregulation of $\mathrm{p} 21$ promotes cell cycle progression, thus these results suggest that the overexpression of miR-512-3p may promote cell cycle progression by inhibiting p21 $(38,39)$.

GO category and KEGG pathway analyses. To obtain valuable insights into the potential mechanisms of miR-512-3p, a bioinformatics analysis was performed to identify the target genes of miR-512-3p using starBase (40). starBase is a database that combines data from six prediction programs TargetScan (41), PicTar (www.pictar.org/), miRanda 

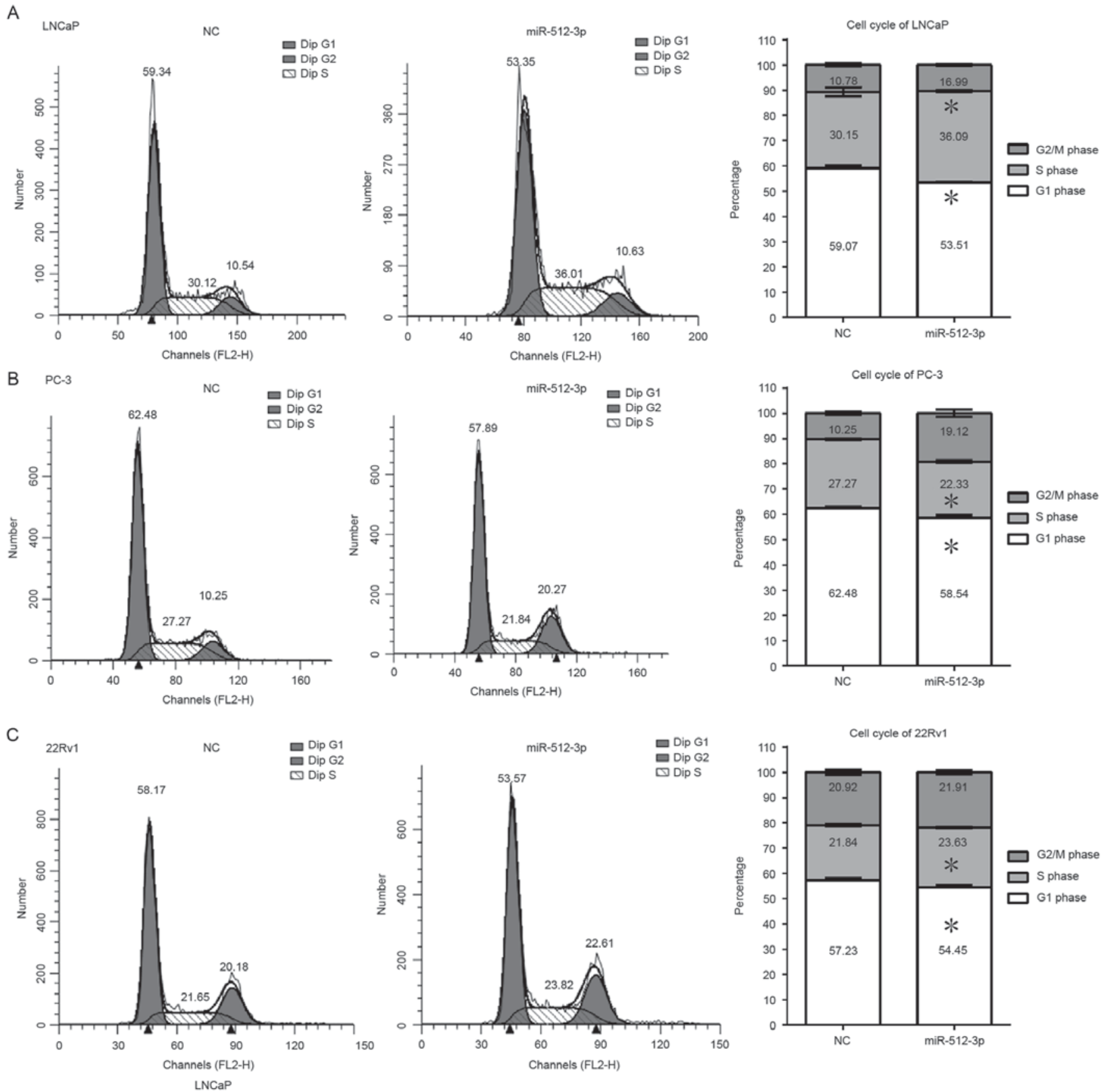

D

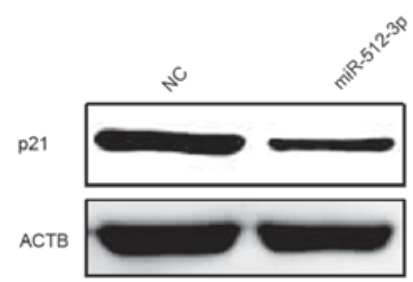

Figure 3. Cell cycle analysis was performed in LNCaP, 22RV1 and PC-3 cells. (A-C) Cells were transfected with a miR-512-3p mimic for $48 \mathrm{~h}$, and were then stained with propidium iodide and evaluated using a FACSCalibur flow cytometer. (D) Protein expression levels of p21 were detected in cells overexpressing miR-512-3p. A western blot assay revealed that overexpression of miR-512-3p inhibited p21 expression. Data are presented as the mean \pm standard deviation $(\mathrm{n}=3)$. "P<0.05 vs. NC. ACTB, $\beta$-actin; miR, microRNA; NC, negative control.

(www.microrna.org/microrna/home.do), PITA (https://genie. weizmann.ac.il/), RNA22 (https://cm.jefferson.edu/rna22/) and CLIP-Seq (www.starbase.sysu.edu.cn/). A total of 663 targets of miR-512-3p were used to perform the KEGG pathway (www.genome.jp/kegg/) and GO category (www.geneontology. org/) analyses using MAS 3.0 system (http://bioinfo.capitalbio. $\mathrm{com} / \mathrm{mas} 3 /)$. The results revealed that miR-512-3p may affect numerous biological processes, including cell adhesion, cell proliferation, cell cycle and apoptosis (Fig. 4A). Pathway enrichment analysis demonstrated that miR-512-3p 
A

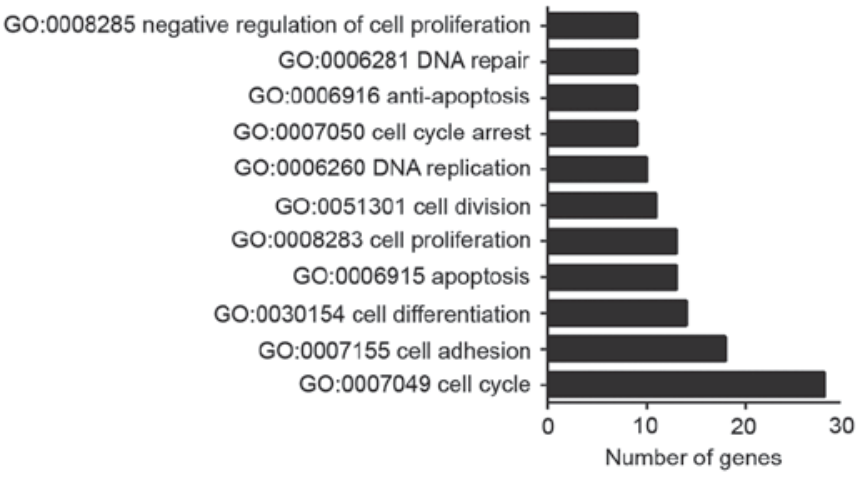

B

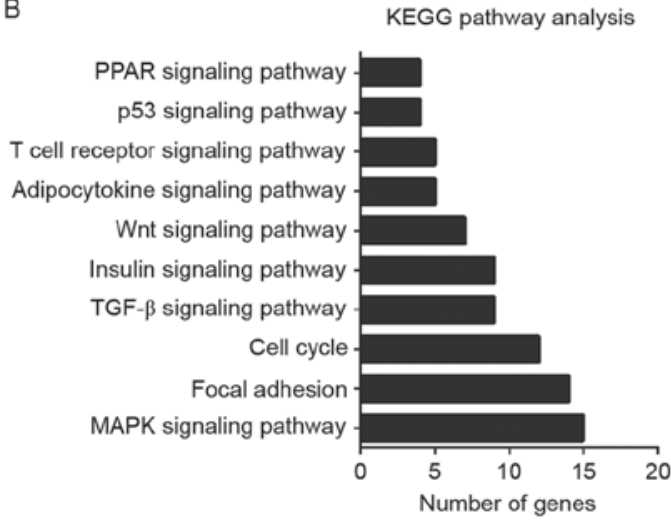

C

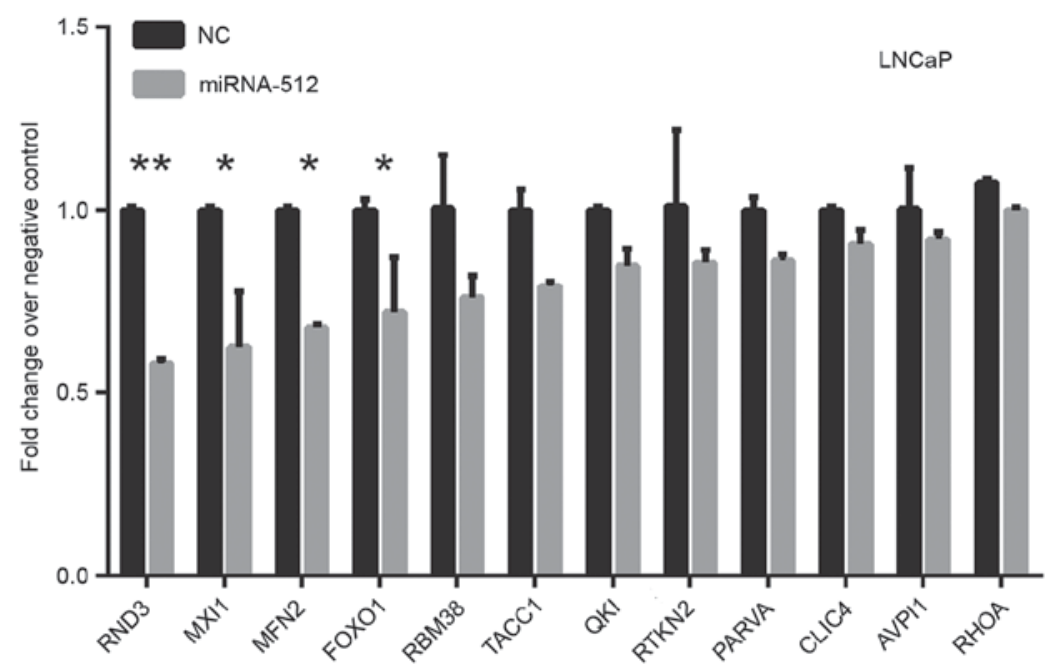

Figure 4. (A) Biological processes and (B) KEGG pathway analyses of miR-512-3p. (C) mRNA expression levels of numerous pathway-related genes following overexpression of miR-512-3p. ${ }^{*} \mathrm{P}<0.05$ and ${ }^{* *} \mathrm{P}<0.01$ vs. NC. AVPI1, arginine vasopressin induced 1; CLIC4, chloride intracellular channel 4; FOXO1, forkhead box O1; GO, Gene Ontology; KEGG, Kyoto Encyclopedia of Genes and Genomes; MAPK, mitogen-activated protein kinase; miR, microRNA; MXI1, MX dynamin like GTPase 1; NC, negative control; PARVA, parvin $\alpha$; PPAR, peroxisome proliferator-activated receptor; QKI, QKI, KH domain containing RNA binding; RBM38, RNA binding motif protein 38; RND3, Rho family GTPase 3; TACC1, transforming coiled-coil containing protein 1; TGF, transforming growth factor; MFN2, mitofusin 2; RHOA, Ras homolog family member A; RTKN2, rhotekin 2.

predominantly participated in the mitogen-activated protein kinase (MAPK) signaling pathway, focal adhesion, cell cycle and transforming growth factor (TGF)- $\beta$ pathway (Fig. 4B). To further validate these findings the present study detected the expression of numerous pathway-associated genes using RT-qPCR. LNCaP cell lines retain characteristics associated with early androgen-dependent molecular biology and tumor cytology (42), and LNCaP is one of the most commonly used cell lines in the PCa research field (43). Thus, LNCaP cell selected for further validation. Overexpression of miR-512-3p was able to significantly reduce the expression levels of Rho family GTPase 3, MAX interactor 1, dimerization protein, MFN2 and forkhead box O1 (Fig. 4C).

\section{Discussion}

PCa is a leading cause of cancer-associated mortality in men worldwide; however, the precise molecular mechanisms underlying the progression of PCa remain unclear. Numerous studies have revealed that miRNAs regulate several biological processes in $\mathrm{PCa}$, including proliferation, cell cycle progression and metastasis (44-47). miRNA expression profiles provide valuable insights into the molecular mechanisms of PCa, and may be used to identify novel biomarkers of PCa. The present study analyzed two publicly available gene expression datasets and screened differentially expressed miRNAs in PCa.

A total of 17 miRNAs were downregulated and 17 miRNAs were overexpressed in PCa samples compared with normal controls in both datasets. The present study primarily focused on the 17 upregulated miRNAs (miR-106b, miR-93, miR-148a, miR-25, miR-375, miR-130b, miR-512-3p, miR-18a, miR-518c*, miR-7, miR-95, miR-96, miR-32, miR-663, miR-182, miR-183 and miR-153) as putative biomarkers. The majority of these miRNAs have been reported to be involved in the carcinogenesis of PCa (27-37).

The present study revealed that miR-512-3p was significantly upregulated in PCa compared with in normal tissues; however, to the best of our knowledge, the molecular functions of miR-512-3p in PCa have yet to be reported in PCa. In lung adenocarcinoma, $\mathrm{miR}-512-3 \mathrm{p}$ has been reported to act as a tumor suppressor that inhibits cell adhesion, migration and invasion of NSCLC cells (23). However, the roles of miR-512-3p in PCa remain unclear. The present study demonstrated that overexpression of miR-512-3p promoted PCa cell proliferation and reduced $\mathrm{G}_{1}$ phase cell cycle arrest in PCa. The results indicated that miR-512-3p may act as an 
oncogene in $\mathrm{PCa}$ and may serve varying roles in different types of cancers.

To obtain valuable insights into the potential mechanisms of miR-512-3p, a bioinformatics analysis was conducted to identify miR-512-3p target genes using starBase (40). A total of 663 targets of miR-512-3p were used to perform KEGG pathway and GO category analyses. The results revealed that miR-512-3p may affect numerous biological processes, including cell adhesion, cell proliferation, cell cycle and apoptosis. Pathway enrichment analyses demonstrated that miR-512-3p was associated with the MAPK signaling pathway, focal adhesion, cell cycle and TGF- $\beta$ pathway. Further validation revealed that overexpression of miR-512-3p significantly reduced the expression levels of RND3, MXI1, MFN2 and FOXO1. These results suggest that miR-512-3p may serve an important role in the regulation of $\mathrm{PCa}$ progression by regulating several genes, including RND3, MXI1, MFN2 and FOXO1.

In conclusion, the present study analyzed two publicly available gene expression datasets, and screened differentially expressed miRNAs in $\mathrm{PCa}$. The results demonstrated that miR-512-3p may promote PCa cell proliferation and cell cycle progression, thus suggesting that miR-512-3p may be considered a potential diagnostic and therapeutic target of PCa.

\section{References}

1. Torre LA, Bray F, Siegel RL, Ferlay J, Lortet-Tieulent J and Jemal A: Global cancer statistics, 2012. CA Cancer J Clin 65: 87-108, 2015

2. Siegel RL, Miller KD and Jemal A: Cancer statistics, 2015. CA Cancer J Clin 65: 5-29, 2015

3. Wang D, Ding L, Wang L, Zhao Y, Sun Z, Karnes RJ, Zhang J and Huang H: LncRNA MALAT1 enhances oncogenic activities of EZH2 in castration-resistant prostate cancer. Oncotarget 6: 41045-41055, 2015.

4. Misawa A, Takayama K, Urano T and Inoue S: Androgen-induced long noncoding RNA (lncRNA) SOCS2-AS1 promotes cell growth and inhibits apoptosis in prostate Cancer Cells. J Biol Chem 291: 17861-17880, 2016.

5. Işın M, Uysaler E, Özgür E, Köseoğlu H, Şanlı Ö, Yücel ÖB, Gezer U and Dalay N: Exosomal IncRNA-p21 levels may help to distinguish prostate cancer from benign disease. Front Genet 6 : $168,2015$.

6. Zhu M, Chen Q, Liu X, Sun Q, Zhao X, Deng R, Wang Y, Huang J, Xu M, Yan J and Yu J: lncRNA H19/miR-675 axis represses prostate cancer metastasis by targeting TGFBI. FEBS J 281: 3766-3775, 2014.

7. Zhang JX, Zhai JF, Yang XT and Wang J: MicroRNA-132 inhibits migration, invasion and epithelial-mesenchymal transition by regulating TGF $\beta 1 / \mathrm{Smad} 2$ in human non-small cell lung cancer. Eur Rev Med Pharmacol Sci 20: 3793-3801, 2016.

8. Zhou Q, Zhu Y, Wei X, Zhou J, Chang L, Sui H, Han Y, Piao D, Sha R and Bai Y: MiR-590-5p inhibits colorectal cancer angiogenesis and metastasis by regulating nuclear factor 90/vascular endothelial growth factor A axis. Cell Death Dis 7: e2413, 2016.

9. Cui Z and Hu Y: MicroRNA-124 suppresses Slug-mediated lung cancer metastasis. Eur Rev Med Pharmacol Sci 20: 3802-3811, 2016.

10. Zhi Y, Pan J, Shen W, He P, Zheng J, Zhou X, Lu G, Chen Z and Zhou Z: Ginkgolide B inhibits human bladder cancer cell migration and invasion through MicroRNA-223-3p. Cell Physiol Biochem 39: 1787-1794, 2016.

11. Zuo J, Wang D, Shen H, Liu F, Han J and Zhang X: MicroRNA-153 inhibits tumor progression in esophageal squamous cell carcinoma by targeting SNAI1. Tumour Biol Oct 13, 2016 (Epub ahead of print)

12. Mo W, Zhang J, Li X, Meng D, Gao Y, Yang S, Wan X, Zhou C, Guo F, Huang Y, et al: Identification of novel AR-targeted microRNAs mediating androgen signalling through critical pathways to regulate cell viability in prostate cancer. PLoS One 8: e56592, 2013.
13. Fletcher CE, Dart DA, Sita-Lumsden A, Cheng H, Rennie PS and Bevan CL: Androgen-regulated processing of the oncomir miR-27a, which targets prohibitin in prostate cancer. Hum Mol Genet 21: 3112-3127, 2012.

14. Kroiss A, Vincent S, Decaussin-Petrucci M, Meugnier E, Viallet J, Ruffion A, Chalmel F, Samarut J and Allioli N: Androgenregulated microRNA-135a decreases prostate cancer cell migration and invasion through downregulating ROCK1 and ROCK2. Oncogene 34: 2846-2855, 2015.

15. Hua X, Xiao Y, Pan W, Li M, Huang X, Liao Z, Xian Q and Yu L: miR-186 inhibits cell proliferation of prostate cancer by targeting GOLPH3. Am J Cancer Res 6: 1650-1660, 2016.

16. Wang Y, Shao N, Mao X, Zhu M, Fan W, Shen Z, Xiao R, Wang C, Bao W, Xu X, et al: MiR-4638-5p inhibits castration resistance of prostate cancer through repressing Kidins220 expression and PI3K/AKT pathway activity. Oncotarget 7: 47444-47464, 2016.

17. Shi XB, Ma AH, Xue L, Li M, Nguyen HG, Yang JC, Tepper CG, Gandour-Edwards R, Evans CP, Kung HJ and deVere White RW: miR-124 and androgen receptor signaling inhibitors repress prostate cancer growth by downregulating androgen receptor splice variants, EZH2, and Src. Cancer Res 75: 5309-5317, 2015.

18. Qin W, Pan Y, Zheng X, Li D, Bu J, Xu C, Tang J, Cui R, Lin P and Yu X: MicroRNA-124 regulates TGF- $\alpha$-induced epithelial-mesenchymal transition in human prostate cancer cells. Int J Oncol 45: 1225-1231, 2014.

19. Lü L, Yuan JD, Cao ZL, Huang T, Zhang CH, Wang L and Zeng FQ: MiR-124 suppresses the proliferation of human prostate cancer PC 3 cells by targeting PKM2. Zhonghua Nan Ke Xue 20: 495-499, 2014 (In Chinese).

20. Sato S, Katsushima K, Shinjo K, Hatanaka A, Ohka F, Suzuki S, Naiki-Ito A, Soga N, Takahashi S and Kondo Y: Histone deacetylase inhibition in prostate cancer triggers miR-320-mediated suppression of the androgen receptor. Cancer Res 76: 4192-4204, 2016.

21. Hsieh IS, Chang KC, Tsai YT, Ke JY, Lu PJ, Lee KH, Yeh SD, Hong TM and Chen YL: MicroRNA-320 suppresses the stem cell-like characteristics of prostate cancer cells by downregulating the Wnt/beta-catenin signaling pathway. Carcinogenesis 34: 530-538, 2013

22. Chen F, Zhu HH, Zhou LF, Wu SS, Wang J and Chen Z: Inhibition of c-FLIP expression by miR-512-3p contributes to Taxol-induced apoptosis in hepatocellular carcinoma cells Oncol Rep 23: 1457-1462, 2010.

23. Zhu X, Gao G, Chu K, Yang X, Ren S, Li Y, Wu H, Huang Y and Zhou C: Inhibition of RAC1-GEF DOCK3 by miR-512-3p contributes to suppression of metastasis in non-small cell lung cancer. Int J Biochem Cell Biol 61: 103-114, 2015.

24. Schaefer A, Jung M, Mollenkopf HJ, Wagner I, Stephan C, Jentzmik F, Miller K, Lein M, Kristiansen G and Jung K: Diagnostic and prognostic implications of microRNA profiling in prostate carcinoma. Int J Cancer 126: 1166-1176, 2010.

25. Taylor BS, Schultz N, Hieronymus H, Gopalan A, Xiao Y, Carver BS, Arora VK, Kaushik P, Cerami E, Reva B, et al: Integrative genomic profiling of human prostate cancer. Cancer Cell 18: 11-22, 2010.

26. Livak KJ and Schmittgen TD: Analysis of relative gene expression data using real-time quantitative PCR and the 2(-Delta Delta C(T)) method. Methods 25: 402-408, 2001.

27. Choi N, Park J, Lee JS, Yoe J, Park GY, Kim E, Jeon H, Cho YM, Roh TY and Lee Y: miR-93/miR-106b/miR-375-CIC-CRABP1: A novel regulatory axis in prostate cancer progression. Oncotarget 6: 23533-23547, 2015.

28. Wang Y, Lieberman R, Pan J, Zhang Q, Du M, Zhang P, Nevalainen M, Kohli M, Shenoy NK, Meng H, et al: miR-375 induces docetaxel resistance in prostate cancer by targeting SEC23A and YAP1. Mol Cancer 15: 70, 2016.

29. Xu L, Zhong J, Guo B, Zhu Q, Liang H, Wen N, Yun W and Zhang L: miR-96 promotes the growth of prostate carcinoma cells by suppressing MTSS1. Tumour Biol 37: 12023-12032, 2016.

30. Jalava SE, Urbanucci A, Latonen L, Waltering KK, Sahu B, Jänne OA, SeppäläJ,LähdesmäkiH, Tammela TL and Visakorpi T: Androgen-regulated miR-32 targets BTG2 and is overexpressed in castration-resistant prostate cancer. Oncogene 31: 4460-4471, 2012.

31. Jiao L, Deng Z, Xu C, Yu Y, Li Y, Yang C, Chen J, Liu Z, Huang G, Li LC and Sun Y: miR-663 induces castration-resistant prostate cancer transformation and predicts clinical recurrence. J Cell Physiol 229: 834-844, 2014. 
32. Wallis CJ, Gordanpour A, Bendavid JS, Sugar L, Nam RK and Seth A: MiR-182 is associated with growth, migration and invasion in prostate cancer via suppression of FOXO1. J Cancer 6: $1295-1305,2015$.

33. Larne O, Östling P, Haflidadóttir BS, Hagman Z, Aakula A Kohonen P, Kallioniemi O, Edsjö A, Bjartell A, Lilja $\mathrm{H}$, et al: miR-183 in prostate cancer cells positively regulates synthesis and serum levels of prostate-specific antigen. Eur Urol 68 : $581-588,2015$.

34. Wu Z, He B, He J and Mao X: Upregulation of miR-153 promotes cell proliferation via downregulation of the PTEN tumor suppressor gene in human prostate cancer. Prostate 73: 596-604, 2013.

35. Murata T, Takayama K, Katayama S, Urano T, Horie-Inoue K, Ikeda K, Takahashi S, Kawazu C, Hasegawa A, Ouchi Y, et al: miR-148a is an androgen-responsive microRNA that promotes LNCaP prostate cell growth by repressing its target CAND1 expression. Prostate Cancer Prostatic Dis 13: 356-361, 2010.

36. Chen Q, Zhao X, Zhang H, Yuan H, Zhu M, Sun Q, Lai X, Wang Y, Huang J, Yan J and Yu J: MiR-130b suppresses prostate cancer metastasis through down-regulation of MMP2. Mol Carcinog 54: 1292-1300, 2015.

37. Ottman R, Levy J, Grizzle WE and Chakrabarti R: The other face of miR-17-92a cluster, exhibiting tumor suppressor effects in prostate cancer. Oncotarget 7: 73739-73753, 2016.

38. Yanagi T, Nagai K, Shimizu H and Matsuzawa SI: Melanoma antigen A12 regulates cell cycle via tumor suppressor p21 expression. Oncotarget 8: 68448-68459, 2017.

39. Prasad R and Katiyar SK: Down-regulation of miRNA-106b inhibits growth of melanoma cells by promoting G1-phase cell cycle arrest and reactivation of $\mathrm{p} 21 / \mathrm{WAF} 1 / \mathrm{Cip} 1$ protein Oncotarget 5: 10636-10649, 2014.
40. Li JH, Liu S, Zhou H, Qu LH and Yang JH: starBase v2.0: Decoding miRNA-ceRNA, miRNA-ncRNA and protein-RNA interaction networks from large-scale CLIP-Seq data. Nucleic Acids Res 42 (Database Issue): D92-D97, 2014

41. Lewis BP, Burge CB and Bartel DP: Conserved seed pairing, often flanked by adenosines, indicates that thousands of human genes are microRNA targets. Cell 120: 15-20, 2005.

42. Horoszewicz JS, Leong SS, Kawinski E, Karr JP, Rosenthal H, Chu TM, Mirand EA and Murphy GP: LNCaP model of human prostatic carcinoma. Cancer Res 43: 1809-1818, 1983.

43. Seim I, Jeffery PL, Thomas PB, Nelson CC and Chopin LK: Whole-genome sequence of the metastatic PC3 and LNCaP human prostate cancer cell lines. G3 (Bethesda) 7: 1731-1741, 2017.

44. Chen W, Liu Y, Chen H, Ning $H$ and Ding K: Loss of miR-449a-caused PrLZ overexpression promotes prostate cancer metastasis. Int J Oncol 51: 435-444, 2017.

45. Zhou Y, Ji Z, Yan W, Zhou Z and Li H: The biological functions and mechanism of miR-212 in prostate cancer proliferation, migration and invasion via targeting Engrailed-2. Oncol Rep 38: 1411-1419, 2017.

46. Lu S, Wang MS, Chen PJ, Ren Q and Bai P: miRNA-186 inhibits prostate cancer cell proliferation and tumor growth by targeting YY1 and CDK6. Exp Ther Med 13: 3309-3314, 2017.

47. Huang YQ, Ling XH, Yuan RQ, Chen ZY, Yang SB, Huang HX, Zhong WD and Qiu SP: miR-30c suppresses prostate cancer survival by targeting the ASF/SF2 splicing factor oncoprotein. Mol Med Rep 16: 2431-2438, 2017. 